\title{
Visual Subfield Progression in Glaucoma Subtypes
}

\author{
Wei-Wen Su $\mathbb{D},{ }^{1}$ Shian-Sen Hsieh, ${ }^{2}$ Shih-Tsung Cheng, ${ }^{3}$ Cheng-Wen Su, ${ }^{3}$ Wei-Chi Wu $\mathbb{D},{ }^{1}$ \\ and Henry Shen-Lih Chen ${ }^{1}{ }^{1}$ \\ ${ }^{1}$ Department of Ophthalmology, Chang Gung Memorial Hospital, Taoyuan, Taiwan \\ ${ }^{2}$ Department of Internal Medicine, Chang Gung Memorial Hospital, Taoyuan, Taiwan \\ ${ }^{3}$ Department of Cardiology, Buddhist Tzu Chi General Hospital Taipei Branch, Xindian, New Taipei City, Taiwan
}

Correspondence should be addressed to Wei-Wen Su; vickysuweiwen@gmail.com

Received 6 August 2017; Accepted 18 January 2018; Published 21 March 2018

Academic Editor: Terri L. Young

Copyright (c) 2018 Wei-Wen Su et al. This is an open access article distributed under the Creative Commons Attribution License, which permits unrestricted use, distribution, and reproduction in any medium, provided the original work is properly cited.

Purpose. To investigate visual field progression pattern and factors associated with progression in patients with primary open-angle glaucoma (POAG), normal-tension glaucoma (NTG), and chronic angle-closure glaucoma (CACG). Methods. The raw data of the 30-2 Humphrey Field Analyzer from glaucoma patients with definite visual field progression were processed with pointwise linear regression (PLR) analysis. The rate of change of retinal threshold sensitivity in the ten glaucoma hemifield test (GHT) zones, the upper and the lower hemifields, and the whole field was evaluated and was correlated with patients' basic demographic data. Results. An average follow-up of $6.94 \pm 2.69$ years that showed the rate of change of visual field threshold sensitivity was correlated with the peak posttreatment intraocular pressure (IOP) and the long-term IOP fluctuations in all GHT zones except in the inferior arcuate area. The baseline IOP, the trough posttreatment IOP, the refractive status, and the CCT were not correlated with VF progression. Conclusion. The rate of visual field progression was correlated with the peak posttreatment IOP and the long-term IOP fluctuation but with subfield differences.

\section{Introduction}

Glaucoma, one of the leading causes of blindness worldwide, is a group of ocular diseases characterized by a specific pattern of abnormalities of the optic nerve head and the retinal nerve fiber layer, with corresponding visual field loss. The key feature of glaucoma pathogenesis is progressive degeneration of retinal ganglion cells that leads to irreversible optic nerve damage and ultimately vision loss. Accurate and timely detection and quantification of disease progression are crucial to ensure appropriate clinical management to preserve long-term vision.

The Humphrey Field Analyzer (HFA; Carl Zeiss Meditec, Dublin, CA, USA) is a commonly used measurement to detect the extent of functional loss and disease worsening in patients with glaucoma. Several methods have been applied to identify visual field deterioration [1-4], categorized as either event-based or trend-based analyses. The event-based analysis compares the differences between baseline and follow-up examinations and matches to the test-retest variability of the reference sample of patients. Progression is flagged when the observed changes exceeds the predefined test-retest limits [5]. The trend-based analysis detects progression as changes over time in serial measurements and provides useful information in the long term, since glaucoma is a progressive disease. The mean deviation (MD) slope and the visual field index (VFI) slope of the Humphrey Field Analyzer are typical global indexes for determining and predicting glaucoma progression; both are very helpful in clinical practice [6]. However, early glaucoma changes are usually trivial and localized, which cannot be detected efficiently by the global indexes. Pointwise linear regression (PLR) that reveals the regression of retinal threshold sensitivity versus time at each test location is another way to evaluate visual field progression. A test location is considered to be progressing if it fulfills specified slope and significance criteria. Visual field progression is defined according to the number of test locations showing the given magnitude of slope and statistical significance $[5,7,8]$. PLR has been 


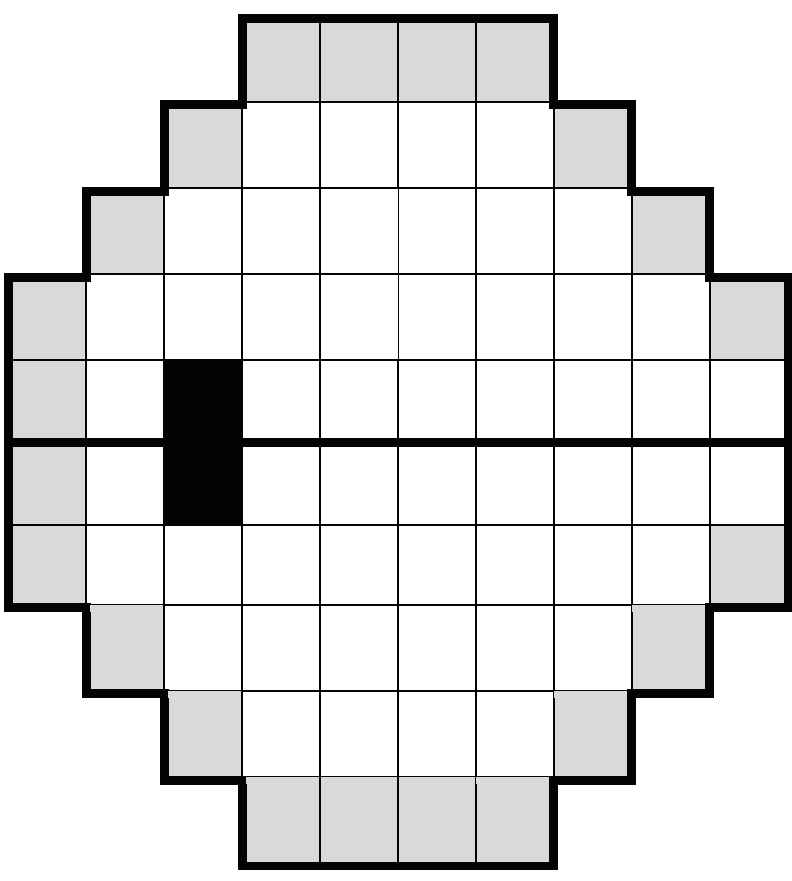

(a)

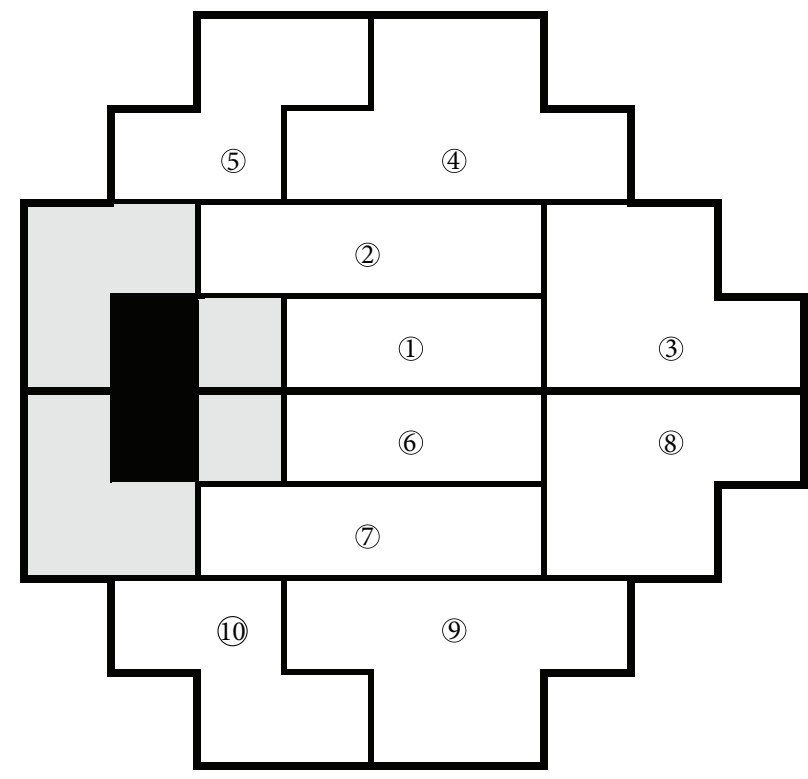

(b)

FIgURE 1: Location of (a) the 52 test points and (b) the 10 GHT zones of the 30-2 SITA program enrolled for pointwise linear regression analysis. Cells in gray and black are edge points and points corresponding to the blind spot that were removed from calculation. Digit in circle indicates the GHT zone number. GHT: glaucoma hemifield test.

used frequently in research settings to detect visual field progression [9-11].

Randomized clinical trials had identified intraocular pressure (IOP) as a major risk factor for the development and progression of glaucoma [12-17]. Refractive status and central corneal thickness (CCT) had also been reported to be predictive for the development or progression of glaucoma [18-22]. Different glaucoma subtypes may progress at different rates $[23,24]$, and there are subfield differences in response to common causative factors [25]. The primary objective of this report is to evaluate the visual subfield progression rate in glaucoma subtypes and to identify clinical factors associated with their progression.

\section{Materials and Methods}

2.1. Participants. The raw data of retinal sensitivity (in $\mathrm{dB}$ ) from the Humphrey Field Analyzer 30-2 Swedish interactive threshold algorithm (SITA) program (Carl Zeiss Meditec, Dublin, CA, USA) between 2001 and 2014 were extracted for analysis. Visual fields with poor reliabilities, defined as fixation losses $>20 \%$ or false positive errors $>15 \%$ or false negative errors $>33 \%$, were excluded from analysis. For reliable tests, those with five or more follow-ups were enrolled for progression analysis. After eliminating edge points (except the two nasal-most points across the horizontal midline) and the two points corresponding to the blind spot, the rate of change of retinal threshold sensitivities in each of the 52 test points (Figure 1(a)) was calculated with pointwise linear regression. Progressive fields were defined as having two or more significant progressive points in the same hemifield; each had a slope of sensitivity of less than $-1.0 \mathrm{~dB}$ /year at $P<0.01$, which was a frequently applied criterion for PLR analysis [26]. Eyes with definite visual field progression were recruited for further analysis. The rate of change of VF threshold sensitivity in each 52 test points, the 10 glaucoma hemifield test (GHT) zones (clusters of locations based on GHT) (Figure 1(b)), the superior and the inferior hemifield, and the whole field were investigated.

The recruited eyes should present typical glaucomatous disc changes and corresponding visual field losses. The interval between tests were generally every 6 months. Eyes with previous ocular trauma or ocular diseases other than glaucoma were excluded. The enrolled eyes were classified into 3 groups according to the angle structure and the baseline IOP. The primary open-angle glaucoma (POAG) group had open anterior chamber angle on gonioscopy, and baseline IOP exceeded $21 \mathrm{mmHg}$. The normal-tension glaucoma (NTG) group was identical to POAG except that the baseline IOP never exceeded $21 \mathrm{mmHg}$ measured at varying intervals between $8 \mathrm{AM}$ to $5 \mathrm{PM}$. The chronic angle-closure glaucoma (CACG) group had closed anterior chamber angles despite patent iridotomies. All glaucoma patients were under medical treatment.

The demographic information including the patients' age of onset, gender, baseline IOP, peak and trough posttreatment IOP during the entire follow-up period (maxIOP and minIOP), long-term IOP fluctuation (IOPf, the difference between the peak and trough IOP), central corneal thickness (CCT), refractive status, baseline and final MD, 
TABLE 1: Basic demographics between the three glaucoma subtypes.

\begin{tabular}{|c|c|c|c|c|}
\hline & POAG $(n=29)$ & NTG $(n=27)$ & CACG $(n=9)$ & $P$ value \\
\hline Number of progressive points & $8.45 \pm 7.99$ & $5.37 \pm 3.14$ & $6.67 \pm 5.36$ & 0.171 \\
\hline $\mathrm{M}: \mathrm{F}$ & $20: 9$ & $15: 12$ & $5: 4$ & 0.556 \\
\hline Age of onset & $41.52 \pm 12.69$ & $50.16 \pm 10.97$ & $61.02 \pm 5.41$ & $<0.001$ \\
\hline F/u years & $7.28 \pm 2.76$ & $6.37 \pm 2.87$ & $7.53 \pm 1.58$ & 0.353 \\
\hline Baseline IOP (mmHg) & $24.77 \pm 5.80$ & $14.54 \pm 3.20$ & $20.97 \pm 6.95$ & $<0.001$ \\
\hline IOP $\min (\mathrm{mmHg})$ & $12.02 \pm 3.15$ & $9.96 \pm 1.85$ & $9.62 \pm 1.98$ & 0.005 \\
\hline IOP $\max (\mathrm{mmHg})$ & $26.05 \pm 8.72$ & $16.53 \pm 2.57$ & $22.28 \pm 2.19$ & $<0.001$ \\
\hline IOP fluctuation (mmHg) & $14.03 \pm 9.23$ & $6.57 \pm 2.07$ & $12.66 \pm 1.59$ & $<0.001$ \\
\hline $\mathrm{CCT}(\mu \mathrm{m})$ & $554.56 \pm 37.11$ & $532.69 \pm 37.12$ & $520.00 \pm 34.84$ & 0.028 \\
\hline SE (D) & $-6.73 \pm 4.74$ & $-4.26 \pm 3.97$ & $+0.06 \pm 2.08$ & $<0.001$ \\
\hline MD baseline (dB) & $-9.51 \pm 7.28$ & $-6.42 \pm 6.34$ & $-10.83 \pm 7.44$ & 0.140 \\
\hline MD final $(\mathrm{dB})$ & $-15.08 \pm 9.01$ & $-10.97 \pm 7.12$ & $-20.86 \pm 7.25$ & 0.007 \\
\hline
\end{tabular}

and pattern standard deviation (PSD) of the visual field tests was collected for correlation analyses. The study followed the tenets of the Declaration of Helsinki and was approved by the Institutional Review Board of Chang Gung Memorial Hospital.

2.2. Statistics. Data were expressed as mean \pm standard deviation (SD) for continuous variables and percentage for categorical variables. The computational statistical environment R (http://www.r-project.org) was used to carry out large-scale pointwise linear regression analyses. For subtypes of glaucoma, continuous variables were compared among the POAG, the NTG, and the CACG group by one-way analysis of variance (ANOVA) followed by Bonferroni multiple comparison test. For relationship between parameters and visual field progression in each GHT zones and hemifields, the Pearson correlation coefficient was calculated. A $P<0.05$ was considered statistically significant.

\section{Results}

Sixty-five eyes from 65 individuals were enrolled in the study, among which 29 were POAG, 27 were NTG, and 9 were CACG. Table 1 lists the basic demographics of the three groups. Patients with POAG were younger, excessively myopic, and had thicker central corneal thickness (CCT). Patients with CACG were older, more hyperopic, and had thinner CCT. Patients with NTG were older at onset than patients with POAG and were less myopic (though statistically insignificant, $P=0.09$ ). Greater IOP fluctuations were noted in the POAG and the CACG groups. The baseline MD of the visual field did not differ in the three groups. Figure 2 shows the rate of change of VF threshold sensitivity in the $10 \mathrm{GHT}$ zones, the superior and the inferior hemifields, and the whole field. An averaged follow-up of $6.94 \pm 2.69$ years showed a faster progression in the superior hemifield in patients with POAG and CACG. The NTG patients demonstrated equal but slightly faster progression in the inferior hemifields.

The age of onset, CCT, refractive status, baseline IOP, and minIOP were not associated with glaucoma progression.
The rate of progression was associated with maxIOP and IOPf in all GHT zones except zone 7, zone 8, and zone 9 (Figure 3). The progression was not correlated with the baseline MD but mildly with the final MD.

\section{Discussion}

In the current study, the average annual progression rate was $-1.00 \pm 0.94 \mathrm{~dB}$ in POAG, $-0.85 \pm 0.41 \mathrm{~dB}$ in NTG, and $-1.12 \pm 0.49 \mathrm{~dB}$ in CACG. Compared to previous investigations, the OHTS reported rate of change in POAG eyes was $-0.26 \pm 0.36 \mathrm{~dB} /$ year [27], the EMGT reported between -0.36 to $-1.31 \mathrm{~dB} /$ year [28], and the CNTGSG reported between -0.41 to $-0.90 \mathrm{~dB} /$ year [29]. Studies from Japan showed the progression rates in treated NTG patients were between -0.1 and $-0.35 \mathrm{~dB} /$ year $[22,30,31]$. The various results among different study groups suggested that high interpatient variability exists in visual field progression.

Myopia had been identified as a risk factor for the development and progression of POAG [21, 32, 33]. Visual field defects worsened with the increase of myopia in patients with POAG, and severe myopia can be a significant risk factor for subsequent visual field loss [34, 35]. On the other hand, some studies showed that the extent of myopia was a significant positive prognostic factor for glaucoma progression $[22,36]$. Araie et al. reported that the less extent of myopia was a significant risk factor for visual field progression in patients with NTG [37]. In the current study, the degree of myopia was not correlated with VF progression in all the GHT subfields. The extent to which myopia affects the progression of OAG remains to be clarified.

CCT had also been identified as a risk factor for glaucoma. Correlation between thinner CCT and progressive visual field loss had been reported $[15,38]$, which might be independent of IOP [39]. In patients with ocular hypertension, the OHTS demonstrated that CCT is an important and independent risk factor for progression to initial glaucoma damage [14]. Visual field progression was significantly associated with thinner CCT both in patients with POAG 


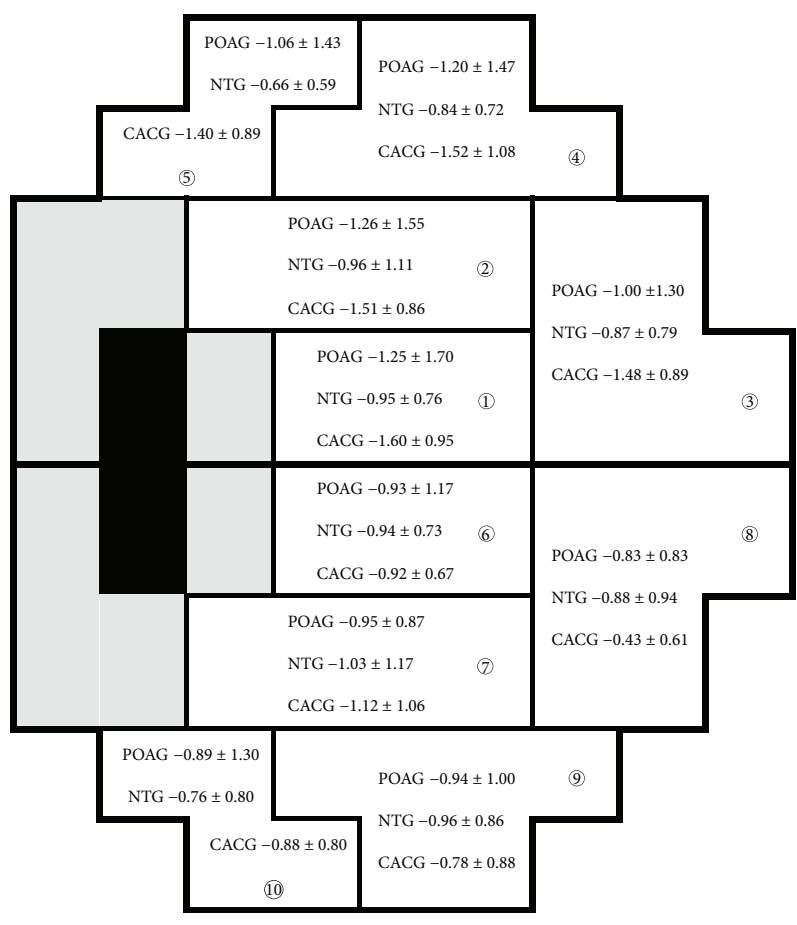

(a)

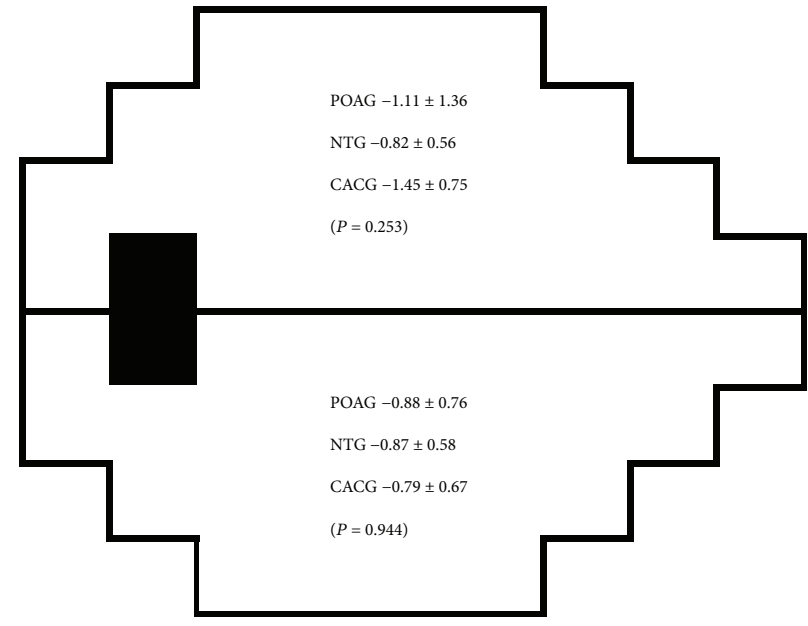

(b)

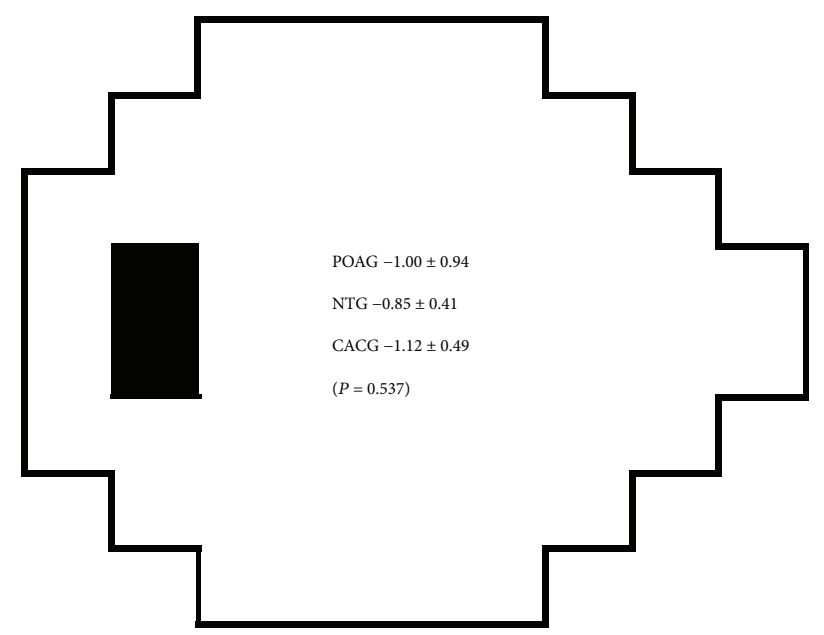

(c)

FIgURE 2: The annual regression slope of VF threshold sensitivity (dB/year) of (a) the 10 GHT zones, (b) the superior and the inferior hemifields, and (c) the whole field in the three glaucoma subtypes. Digit in circle indicates the GHT zone number.

and NTG $[40,41]$. Conversely, Chauhan et al. reported that once glaucomatous damage was present, CCT was unlikely to be a useful predictive index for visual field and optic disc progression [42]. Jonas et al. reported that the amount of glaucomatous optic nerve damage correlated significantly with a thin central cornea, but progression of glaucomatous optic nerve neuropathy was independent of central corneal thickness [43]. Our report demonstrated that although the CCT differed among the three glaucoma subtypes, correlation between CCT and VF progression was not present. Since all our patient had definite visual field progression, CCT may not be a useful correlated factor in this group of patients.

The level of IOP has been shown to be the most important factor for both glaucoma development $[14,44,45]$ and progression $[15,46,47]$. In the EMGT, the magnitude of initial IOP reduction was a major factor influencing outcome [46]. However, in the CNTGSG, the untreated level of intraocular pressure did not affect the rate of untreated disease progression, despite their known influence on prevalence [13]. The current study demonstrated that only the peak posttreatment IOP and the long-term IOP fluctuations were associated with glaucoma progression. Furthermore, subfield analysis showed that the progression in certain area of the visual field, such as the GHT zones 7, 8, 9 (the inferior arcuate area) was not correlated with any parameter of IOP. This finding suggested that the rate of change in certain visual subfield (the inferior arcuate area) may be less sensitive to IOP changes. Visual field progression in the inferior 


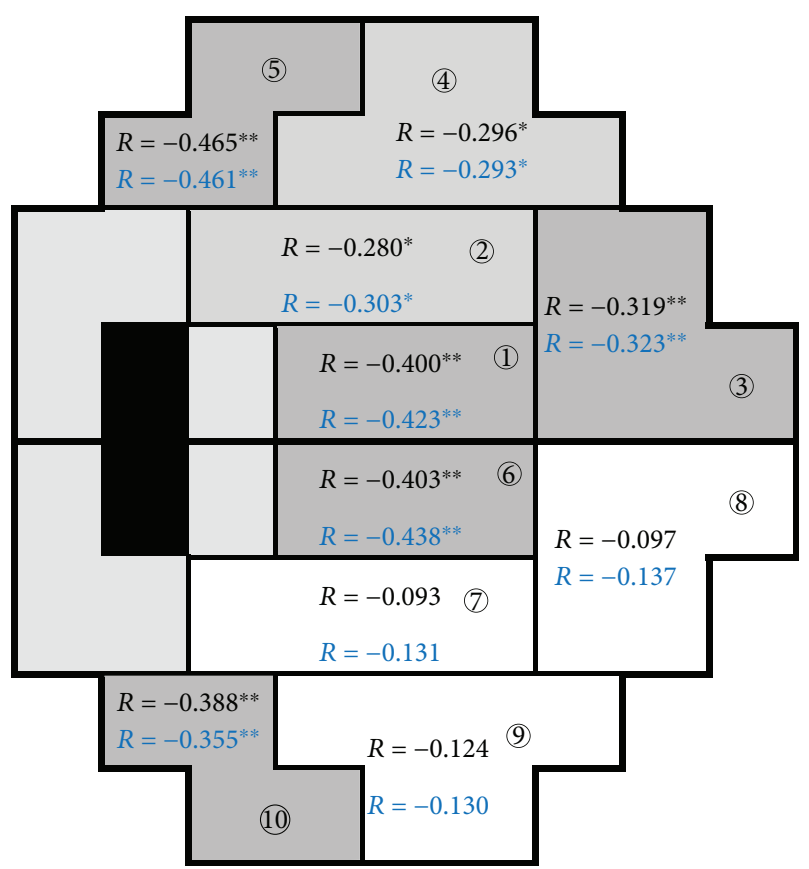

FIGURE 3: The correlation of the maximal posttreatment IOP and the long-term IOP fluctuations with VF progression in the $10 \mathrm{GHT}$ zones. ${ }^{*} P<0.05 ;{ }^{* *} P<0.001$. Digit in circle indicates the GHT zone number. Darker cells indicate a more significant correlation.

peripheral area was reported to be significantly correlated with peripheral vascular endothelial function, suggesting regional vulnerability to vascular dysregulation [48]. The current study further responds to this possibility.

\section{Conclusion}

In general, the superior fields progressed faster than the inferior fields. Among common risk factors for glaucoma, only the peak posttreatment IOP and the long-term IOP fluctuation were correlated with visual field progression. Progression in the inferior arcuate area was not correlated to any IOP parameters, suggesting regional difference in the visual subfield in terms of vulnerability to IOP changes.

\section{Conflicts of Interest}

The authors declare that there is no conflict of interest regarding the publication of this paper.

\section{References}

[1] P. Brusini and C. A. Johnson, "Staging functional damage in glaucoma: review of different classification methods," Survey of Ophthalmology, vol. 52, no. 2, pp. 156-179, 2007.

[2] K. Nouri-Mahdavi, N. Nassiri, A. Giangiacomo, and J. Caprioli, "Detection of visual field progression in glaucoma with standard achromatic perimetry: a review and practical implications," Graefe's Archive for Clinical and Experimental Ophthalmology, vol. 249, no. 11, pp. 1593-1616, 2011.

[3] P. G. Spry and C. A. Johnson, "Identification of progressive glaucomatous visual field loss," Survey of Ophthalmology, vol. 47 , no. 2, pp. 158-173, 2002.
[4] A. A. Aref and D. L. Budenz, "Detecting visual field progression," Ophthalmology, vol. 124, no. 12, pp. S51-S56, 2017.

[5] P. H. Artes and B. C. Chauhan, "Longitudinal changes in the visual field and optic disc in glaucoma," Progress in Retinal and Eye Research, vol. 24, no. 3, pp. 333-354, 2005.

[6] B. Bengtsson, V. M. Patella, and A. Heijl, "Prediction of glaucomatous visual field loss by extrapolation of linear trends," Archives of Ophthalmology, vol. 127, no. 12, pp. 1610-1615, 2009.

[7] T. Krupin, J. M. Liebmann, D. S. Greenfield et al., "The Lowpressure Glaucoma Treatment Study (LoGTS): study design and baseline characteristics of enrolled patients," Ophthalmology, vol. 112, no. 3, pp. 376-385, 2005.

[8] K. Nouri-Mahdavi, J. Caprioli, A. L. Coleman, D. Hoffman, and D. Gaasterland, "Pointwise linear regression for evaluation of visual field outcomes and comparison with the advanced glaucoma intervention study methods," Archives of Ophthalmology, vol. 123, no. 2, pp. 193-199, 2005.

[9] K. A. Baez, A. I. McNaught, J. G. Dowler, D. Poinoosawmy, F. W. Fitzke, and R. A. Hitchings, "Motion detection threshold and field progression in normal tension glaucoma," The British Journal of Ophthalmology, vol. 79, no. 2, pp. 125-128, 1995.

[10] D. P. Crabb, F. W. Fitzke, A. I. McNaught, D. F. Edgar, and R. A. Hitchings, "Improving the prediction of visual field progression in glaucoma using spatial processing," Ophthalmology, vol. 104, no. 3, pp. 517-524, 1997.

[11] J. B. Jonas, P. Martus, F. K. Horn, A. Ju"nemann, M. Korth, and W. M. Budde, "Predictive factors of the optic nerve head for development or progression of glaucomatous visual field loss," Investigative Ophthalmology \& Visual Science, vol. 45, no. 8, pp. 2613-2618, 2004.

[12] The AGIS Investigators, "The Advanced Glaucoma Intervention Study (AGIS): 7. the relationship between control of intraocular pressure and visual field deterioration," American Journal of Ophthalmology, vol. 130, no. 4, pp. 429-440, 2000.

[13] S. Drance, D. R. Anderson, M. Schulzer, and Collaborative Normal-Tension Glaucoma Study G, "Risk factors for progression of visual field abnormalities in normal-tension glaucoma," American Journal of Ophthalmology, vol. 131, no. 6, pp. 699-708, 2001.

[14] M. O. Gordon, J. A. Beiser, J. D. Brandt et al., "The Ocular Hypertension Treatment Study: baseline factors that predict the onset of primary open-angle glaucoma," Archives of Ophthalmology, vol. 120, no. 6, pp. 714-720, 2002.

[15] M. C. Leske, A. Heijl, L. Hyman et al., "Predictors of long-term progression in the early manifest glaucoma trial," Ophthalmology, vol. 114, no. 11, pp. 1965-1972, 2007.

[16] S. Miglior, V. Torri, T. Zeyen et al., "Intercurrent factors associated with the development of open-angle glaucoma in the European Glaucoma Prevention Study," American Journal of Ophthalmology, vol. 144, no. 2, pp. 266-275.e1, 2007.

[17] D. C. Musch, B. W. Gillespie, P. R. Lichter, L. M. Niziol, N. K. Janz, and CIGTS Study Investigators, "Visual field progression in the Collaborative Initial Glaucoma Treatment Study: the impact of treatment and other baseline factors," Ophthalmology, vol. 116, no. 2, pp. 200-207.e1, 2009.

[18] M. Deol, D. A. Taylor, and N. M. Radcliffe, "Corneal hysteresis and its relevance to glaucoma," Current Opinion in Ophthalmology, vol. 26, no. 2, pp. 96-102, 2015.

[19] D. Meira-Freitas, R. Lisboa, A. Tatham et al., "Predicting progression in glaucoma suspects with longitudinal estimates 
of retinal ganglion cell counts," Investigative Ophthalmology \& Visual Science, vol. 54, no. 6, pp. 4174-4183, 2013.

[20] C. V. De Moraes, V. Hill, C. Tello, J. M. Liebmann, and R. Ritch, "Lower corneal hysteresis is associated with more rapid glaucomatous visual field progression," Journal of Glaucoma, vol. 21, no. 4, pp. 209-213, 2012.

[21] M. W. Marcus, M. M. de Vries, F. G. Junoy Montolio, and N. M. Jansonius, "Myopia as a risk factor for open-angle glaucoma: a systematic review and meta-analysis," Ophthalmology, vol. 118, no. 10, pp. 1989-1994.e2, 2011.

[22] R. Sakata, M. Aihara, H. Murata et al., "Contributing factors for progression of visual field loss in normal-tension glaucoma patients with medical treatment," Journal of Glaucoma, vol. 22, no. 3, pp. 250-254, 2013.

[23] C. G. De Moraes, J. M. Liebmann, C. A. Liebmann, R. Susanna Jr., C. Tello, and R. Ritch, "Visual field progression outcomes in glaucoma subtypes," Acta Ophthalmologica, vol. 91, no. 3, pp. 288-293, 2013.

[24] S. Verma, M. E. Nongpiur, E. Atalay et al., "Visual field progression in patients with primary angle-closure glaucoma using pointwise linear regression analysis," Ophthalmology, vol. 124, no. 7, pp. 1065-1071, 2017.

[25] C. Mayama, Y. Suzuki, M. Araie et al., "Myopia and advancedstage open-angle glaucoma," Ophthalmology, vol. 109, no. 11, pp. 2072-2077, 2002.

[26] E. Vesti, C. A. Johnson, and B. C. Chauhan, "Comparison of different methods for detecting glaucomatous visual field progression," Investigative Ophthalmology \& Visual Science, vol. 44, no. 9, pp. 3873-3879, 2003.

[27] S. Demirel, C. G. De Moraes, S. K. Gardiner et al., "The rate of visual field change in the ocular hypertension treatment study," Investigative Ophthalmology \& Visual Science, vol. 53, no. 1, pp. 224-227, 2012.

[28] A. Heijl, B. Bengtsson, L. Hyman, M. C. Leske, and Early Manifest Glaucoma Trial Group, "Natural history of openangle glaucoma," Ophthalmology, vol. 116, no. 12, pp. 22712276, 2009.

[29] D. R. Anderson, S. M. Drance, M. Schulzer, and Collaborative normal-tension glaucoma study G, "Natural history of normal-tension glaucoma," Ophthalmology, vol. 108, no. 2, pp. 247-253, 2001.

[30] K. Inoue, S. Hashida, Y. Tajima, M. Wakakura, J. Inoue, and G. Tomita, "Progression of visual field defect in a normaltension glaucoma patient after laser in situ keratomileusis," Eye, vol. 18, no. 8, pp. 850-853, 2004.

[31] M. Araie, S. Shirato, Y. Yamazaki, Y. Kitazawa, Y. Ohashi, and Nipradilol-Timolol Study Group, "Clinical efficacy of topical nipradilol and timolol on visual field performance in normaltension glaucoma: a multicenter, randomized, double-masked comparative study," Japanese Journal of Ophthalmology, vol. 52, no. 4, pp. 255-264, 2008.

[32] Y. Suzuki, A. Iwase, M. Araie et al., "Risk factors for openangle glaucoma in a Japanese population: the Tajimi Study," Ophthalmology, vol. 113, no. 9, pp. 1613-1617, 2006.

[33] T. Y. Wong, B. E. Klein, R. Klein, M. Knudtson, and K. E. Lee, "Refractive errors, intraocular pressure, and glaucoma in a white population," Ophthalmology, vol. 110, no. 1, pp. 211217, 2003.

[34] A. Perdicchi, M. Iester, G. Scuderi, S. Amodeo, E. M. Medori, and S. M. Recupero, "Visual field damage and progression in glaucomatous myopic eyes," European Journal of Ophthalmology, vol. 17, no. 4, pp. 534-537, 2007.

[35] E. Chihara, X. Liu, J. Dong et al., "Severe myopia as a risk factor for progressive visual field loss in primary open-angle glaucoma," Ophthalmologica, vol. 211, no. 2, pp. 66-71, 1997.

[36] J. Y. Lee, K. R. Sung, S. Han, and J. H. Na, "Effect of myopia on the progression of primary open-angle glaucoma," Investigative Ophthalmology \& Visual Science, vol. 56, no. 3, pp. 17751781, 2015.

[37] M. Araie, S. Shirato, Y. Yamazaki et al., "Risk factors for progression of normal-tension glaucoma under $\beta$-blocker monotherapy," Acta Ophthalmologica, vol. 90, no. 5, pp. e337-e343, 2012.

[38] F. A. Medeiros, P. A. Sample, L. M. Zangwill, C. Bowd, M. Aihara, and R. N. Weinreb, "Corneal thickness as a risk factor for visual field loss in patients with preperimetric glaucomatous optic neuropathy," American Journal of Ophthalmology, vol. 136, no. 5, pp. 805-813, 2003.

[39] T. S. Prata, C. G. De Moraes, C. C. Teng, C. Tello, R. Ritch, and J. M. Liebmann, "Factors affecting rates of visual field progression in glaucoma patients with optic disc hemorrhage," Ophthalmology, vol. 117, no. 1, pp. 24-29, 2010.

[40] J. W. Kim and P. P. Chen, "Central corneal pachymetry and visual field progression in patients with open-angle glaucoma," Ophthalmology, vol. 111, no. 11, pp. 2126-2132, 2004.

[41] E. Erdem, A. Williams, S. D. Kuchar, M. Waisbourd, and G. L. Spaeth, "Long-term ( $>8$ years) evaluation of progression in patients with low-pressure glaucoma," European Journal of Ophthalmology, vol. 25, no. 6, pp. 490-495, 2015.

[42] B. C. Chauhan, D. M. Hutchison, R. P. LeBlanc, P. H. Artes, and M. T. Nicolela, "Central corneal thickness and progression of the visual field and optic disc in glaucoma," The British Journal of Ophthalmology, vol. 89, no. 8, pp. 1008-1012, 2005.

[43] J. B. Jonas, A. Stroux, I. Velten, A. Juenemann, P. Martus, and W. M. Budde, "Central corneal thickness correlated with glaucoma damage and rate of progression," Investigative Ophthalmology \& Visual Science, vol. 46, no. 4, pp. 12691274, 2005.

[44] S. Miglior, T. Zeyen, N. Pfeiffer et al., "Results of the European Glaucoma Prevention Study," Ophthalmology, vol. 112, no. 3, pp. 366-375, 2005.

[45] European Glaucoma Prevention Study (EGPS) Group, S. Miglior, N. Pfeiffer et al., "Predictive factors for open-angle glaucoma among patients with ocular hypertension in the European Glaucoma Prevention Study," Ophthalmology, vol. 114, no. 1, pp. 3-9, 2007.

[46] M. C. Leske, A. Heijl, M. Hussein et al., "Factors for glaucoma progression and the effect of treatment: the early manifest glaucoma trial," Archives of Ophthalmology, vol. 121, no. 1, pp. 48-56, 2003.

[47] S. Oie, K. Ishida, and T. Yamamoto, "Impact of intraocular pressure reduction on visual field progression in normaltension glaucoma followed up over 15 years," Japanese Journal of Ophthalmology, vol. 61, no. 4, pp. 314-323, 2017.

[48] C. H. Liu, W. W. Su, S. S. Shie, S. T. Cheng, C. W. Su, and W. J. Ho, "Association between peripheral vascular endothelial function and progression of open-angle glaucoma," Medicine, vol. 95, no. 10, article e3055, 2016. 


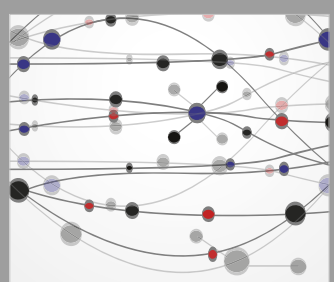

The Scientific World Journal
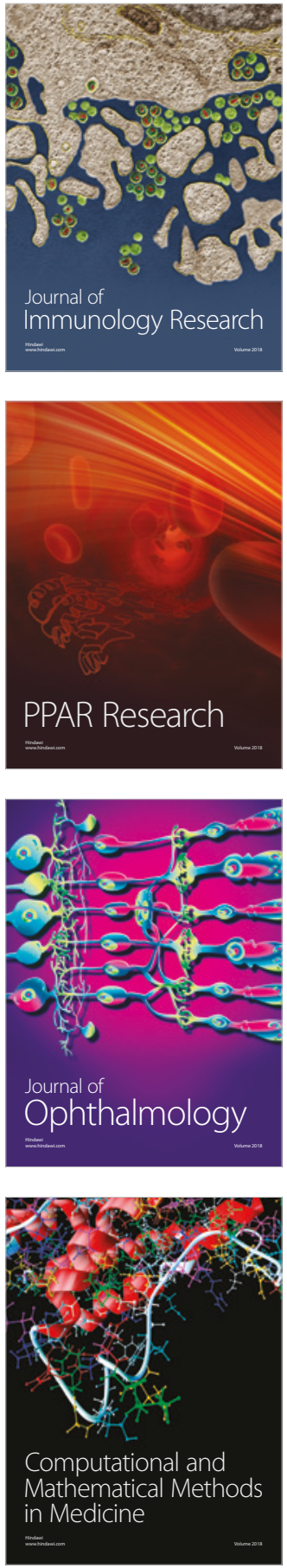

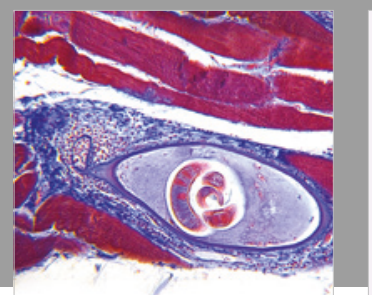

Gastroenterology Research and Practice

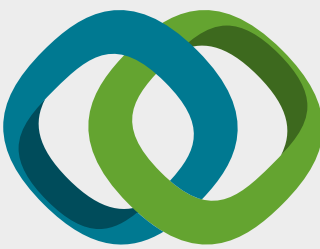

\section{Hindawi}

Submit your manuscripts at

www.hindawi.com
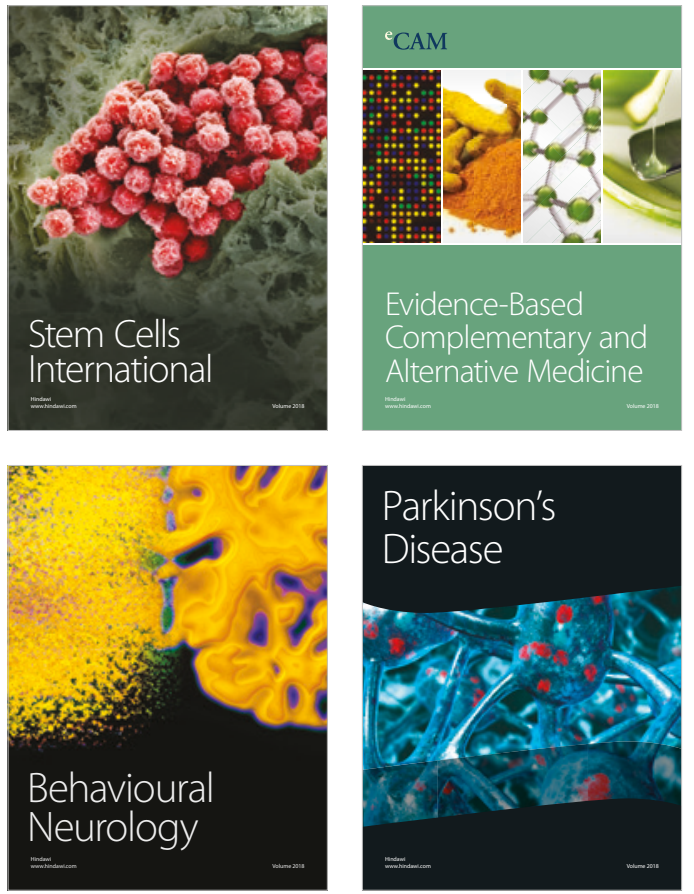

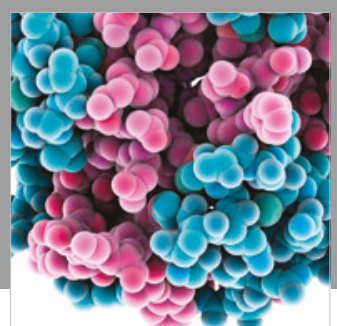

ournal of

Diabetes Research

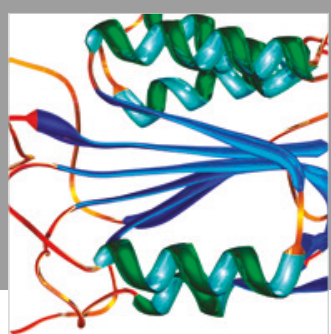

Disease Markers
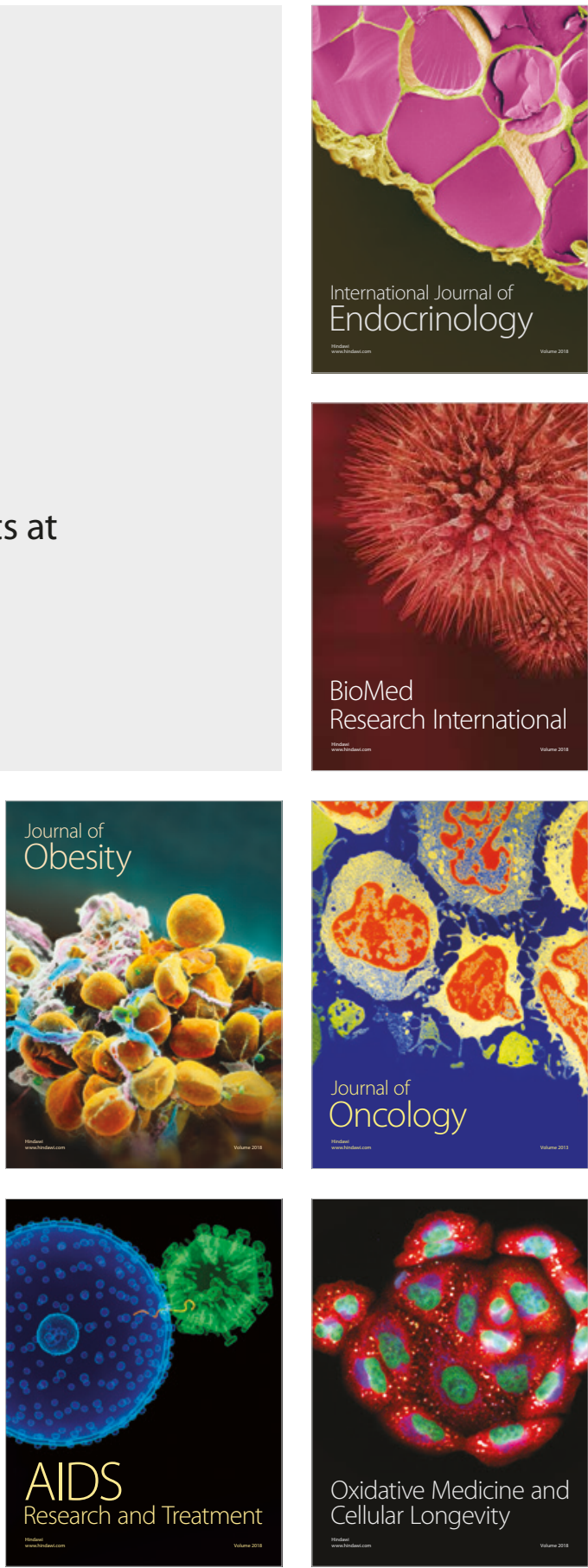